\title{
Role of High Resolution Computed Tomography in the Evaluation of Active Pulmonary Tuberculosis
}

\author{
Authors
Santhosh Raj ${ }^{1}$, Mini M V ${ }^{2}$, Abhilash Babu T G ${ }^{3}$, Mohanan $K^{4}$, Raini KP ${ }^{5}$, Paul V Puthussery ${ }^{6}$
${ }^{1}$ Resident, ${ }^{2,5,6}$ Assistant Professor, ${ }^{3,4}$ Professor \\ Department of Radiodiagnosis, Government Medical College, Thrissur, Kerala, India \\ Corresponding Author \\ Mini M V \\ Assistant Professor of Radiodiagnosis, Government Medical College, Thrissur \\ Email: mini sanjeev72@gmail.com
}

\begin{abstract}
In developing countries, pulmonary tuberculosis is still a common disease particularly among the socioeconomically disadvantaged, elderly and chronically debilitated individuals Chest radiography is the imaging modality of choice in the initial evaluation of patients with suspected pulmonary tuberculosis. HRCT is more sensitive than chest radiography in the detection and characterization of subtle disease in patients with suspected mycobacterial infection. Aim of my study was to assess the HRCT findings of active pulmonary tuberculosis and to find out the association between HRCT findings in active pulmonary tuberculosis with sputum positivity.

Materials and Methods- This is a Prospective Observational study conducted in Radio Diagnosis Department Govt. Medical College, Thrissur from January 2014- June 2015 (18 months). The study sample comprised of 100 patients clinically, radiologically or bacteriologically diagnosed as pulmonary tuberculosis. The findings we took to interpret active pulmonary tuberculosis include centrilobular nodule, "tree in bud" appearance, consolidation, cavity, ground glassing, lymphadenopathy, pleural effusion; parenchymal calcifications, bronchiectasis, miliary nodules and emphysema.

Observation and Conclusion- HRCT findings like centrilobular nodules, "tree in bud", cavitation and mediastinal lymphadenopathy are the best parameters of active pulmonary tuberculosis and there is statistically significant association between these findings and sputum positivity.

Keywords - High Resolution Computed Tomography, pulmonary tuberculosis, Centrilobular Nodules, Tree In Bud appearance.
\end{abstract}

\section{Introduction}

Although the global incidence of $\mathrm{TB}$ has been decreasing, pulmonary TB remains an important cause of morbidity and mortality worldwide. ${ }^{1}$ The most common form of pulmonary tuberculosis in adults is post primary disease or reinfection. Despite all governmental efforts, tuberculosis (TB) remains a public health problem world-wide with almost 9 million new cases each year and almost 2 million TB related deaths world-wide ${ }^{2}$

Delay in diagnosis of active cases of pulmonary TB increases the burden of the disease, and this delay in 
diagnosis is related to many reasons: TB can present clinically and radiologically like many other diseases as pneumonia, malignancy and interstitial lung diseases, the yield of sputum smear is still low and needs few days to get the results3. Culture for mycobacterium TB which is the gold standard in diagnosis of TB needs up to 6 weeks for sure results, even new radiometric cultures need about 2 weeks to give results and not available in every hospital. The delay in diagnosis causes delay in isolation of the patient with more chance for spread of infection and increase in severity of the disease.

Chest radiography is the imaging modality of choice in the initial evaluation of patients with suspected pulmonary tuberculosis and in monitoring response to therapy. HRCT is a useful adjunct to chest X-ray in the diagnosis of tuberculosis and is more sensitive than chest radiography in the detection and characterization of subtle parenchymal disease and mediastinal lymphadenopathy. Although sputum culture for acid fast bacilli is the 'gold standard' for the diagnosis of pulmonary tuberculosis, in patients clinically suspected of having tuberculosis with normal or equivocal radiographic abnormalities, the increased sensitivity of computed tomography may allow prompt diagnosis before results of culture.

\section{Aim of the Study}

1. To assess the findings of active pulmonary tuberculosis on High Resolution Computed Tomography Scan.

2. To find out the association between High Resolution Computed Tomographic findings in active pulmonary tuberculosis with sputum positivity.

\section{Materials \& Methods}

Study design was Prospective Observational study conducted in Radiodiagnosis Department Govt. Medical College, Thrissur. Study period was from January 2014- June 2015 (18 months) and Sample size was taken as 100 . The study sample comprised of 100 patients clinically, radiologically or bacteriologically diagnosed as pulmonary tuberculosis attending the Department of Radio
Diagnosis for HRCT examination irrespective of age and sex. Patients who are reluctant to undergo computed tomography examination and pregnant woman are excluded. Study protocol was submitted to Institutional research committee and Ethical committee and necessary approvals obtained. Data was collected from patients after getting an informed consent. The collected personal information was kept strictly confidential.

\section{Imaging - HRCT}

Patients with active or reinfection tuberculosis diagnosed on clinical, radiological and bacteriological examination findings underwent HRCT examination. HRCT scans were obtained with Siemens Somatom Emotion - 16 CT scanner. Scanning was performed with $1.5 \mathrm{~mm}$ collimation, $120 \mathrm{kvP}, 170 \mathrm{~mA}$, two second scan time, 512x512 matrixes, and a bone algorithm at $10 \mathrm{~mm}$ intervals from the lung apices to the bases. Images were obtained with a $35-40 \mathrm{~cm}$ field of view. Intravenous contrast medium was not administered.

The terms used to interpret HRCT findings in active pulmonary tuberculosis include 1. Centrilobular nodule-well defined lesions 1-4 mm thick, separated by more than $2 \mathrm{~mm}$ from the pleural surface or interlobular septa. 2. "tree in bud appearance"--a branching linear structure with more than one contiguous branching site. In addition, other findings like consolidation, cavity, ground glassing, lymphadenopathy, pleural effusion; parenchymal calcifications, bronchiectasis, miliary nodules and emphysema were also noted.

\section{Statistical Methods}

The data were coded and entered in Microsoft Excel and statistical analysis done using Epi info 7.Baseline data and findings assessed using proportion and $95 \%$ Confidence Interval. Association between HRCT findings and sputum positivity assessed using chi-square test and level of significance assessed using $\mathrm{p}$ value and $95 \%$ Confidence Interval. A value of $\mathrm{p}<0.05$ was considered to be significant. 


\section{Observations}

100 patients were included in the study. Out of that $65(65 \%)$ were males and $35(35 \%)$ were females. Out of the 100 patients most of the patients were in the age group of 51-60 (28\%) and 41-50 (27\%). Only one patient was below the age of 10 and two patients were above the age of 80

The most common HRCT findings detected in the sputum positive group were centrilobular nodules $32(32 \%)$, tree in bud $31(31 \%)$, cavity $22(22 \%)$, lymphadenopathy $23(23 \%)$, ground glass opacity $11(11 \%)$ and consolidation $6(6 \%)$. Other findings like bronchiectasis $68(68 \%)$, pleural effusion 11 (11\%), parenchymal calcification 13 (13\%), emphysema $12(12 \%)$ and miliary nodules $2(2 \%)$ were also noted, mostly in sputum negative patients. Out of the 100 patients sputum AFB was positive in $30(30 \%)$ patients and negative in $70(70 \%)$ patients with $95 \%$ confidence limit of $21-40 \%$. Centrilobular nodules were present in $32(32 \%)$ patients and absent in $68(68 \%)$ patients out of 100 patients included in the study. 95\% confidence limit of 23$42 \%$ noted for the HRCT finding of centrilobular nodule. Centrilobular nodules present in 19 (59.4\%) patients with sputum positivity and 13 (40.6\%) patients with sputum negativity. Chi square value of 19.3365 and $P$ value of 0.000010 noted.

"Tree in bud" pattern were present in $31(31 \%)$ patients and absent in $69(69 \%)$ patients out of 100 patients studied with a $95 \%$ confidence limit of 22 $41 \%$. Tree in bud pattern was detected in 18 patients with sputum positivity and 13 patients with sputum negativity. Chi square value of 16.8503 and $P$ value- 0.000040 .

Out of 100 patients studied, consolidation was present in $6(6 \%)$ patients and absent in $94(94 \%)$ patients with a $95 \%$ confidence limit of $2-12 \%$. Consolidation present in 4 patients with sputum positivity and 2 patients with sputum negativity. Chi square value of 4.0865 and $P$ value of 0.0432 were noted.

Cavities were present in $22(22 \%)$ patients and absent in $78(78 \%)$ patients included in the study with a $95 \%$ confidence limit of $14-31 \%$. Cavity noted in 13 patients with sputum positivity and 9 patients with sputum negativity. Chi square value of 11.3664 and $\mathrm{P}$ value of 0.0007 noted.

Out of 100 patients studied, ground glass opacities were present in $11(11 \%)$ patients and absent in 89 (89\%) patients with a $95 \%$ confidence limit of 5-8\%. Ground glassing was present in 6 patients with sputum positivity and 5 patients with sputum negativity with a Chi square value of 3.5459 and $\mathrm{P}$ value of 0.0596 .

Mediastinal lymphadenopathy was present in 23 (23\%) patients and absent in $77(77 \%)$ patients with a $95 \%$ confidence limit of $15-32 \%$. Lymphadenopathy was present in 13 patients with sputum positivity and 10 patients with sputum negativity with a Chi square value of 10.0051 and $\mathrm{P}$ value of 0.0015 .

Out of 100 patients included in the study, miliary nodules were present in $2(2 \%)$ patients and absent in $98(98 \%)$ patients with $95 \%$ confidence limit of $0.2-7 \%$. Sputum AFB was negative in both cases. Pleural effusion was present in $11(11 \%)$ patients and absent in 89 (89\%) cases with a 95\% confidence limit of 5-18\%. Pleural effusion was present in 5 patients with sputum positivity and 6 patients with sputum negativity. Chi square value of 1.4057 and $\mathrm{P}$ value of 0.2357 noted. Bronchiectasis was present in $68(68 \%)$ patients and absent in 32 (32\%) patients with a $95 \%$ confidence limit of 58$77 \%$. Bronchiectasis was present in 15 patients with sputum positivity and 53 patients with sputum negativity with a Chi square value of 6.3813 and $P$ value of 0.01153 .

Out of 100 patients included in the study, parenchymal calcifications were present in $13(13 \%)$ patients and absent in $87(87 \%)$ patients with a 95\% confidence limit of $7-21 \%$. Parenchymal calcifications were present in none of the patients with sputum positivity. Parenchymal cicatricial emphysema was noted in $12(12 \%)$ patients and absent in $88(88 \%)$ patients with a $95 \%$ confidence interval of 6.36-20.0.None of the patients showed sputum positivity. 


\section{JMSCR Vol||05||Issue||04||Page 20819-20823||April}

Table -1 : HRCT findings in study population $(\mathrm{n}=100)$

\begin{tabular}{|l|c|}
\hline HRCT FINDING & FREQUENCY \\
\hline Centrilobular nodule & 32 \\
\hline Tree in bud & 31 \\
\hline Cavity & 22 \\
\hline Consolidation & 6 \\
\hline Ground glass opacity & 11 \\
\hline Bronchiectasis & 68 \\
\hline Parenchymal calcifications & 13 \\
\hline Lymphadenopathy & 23 \\
\hline Pleural effusion & 11 \\
\hline Miliary nodule & 2 \\
\hline Emphysema & 12 \\
\hline
\end{tabular}

Table - 2: Distribution of various HRCT findings in the study population $(n=100)$

\begin{tabular}{|l|c|c|}
\hline Findings & $\begin{array}{c}\text { Sputum AFB } \\
\text { positive }(\mathrm{n}=30)\end{array}$ & $\begin{array}{c}\text { Sputum AFB } \\
\text { negative }(\mathrm{n}=70)\end{array}$ \\
\hline Centrilobular nodule & 19 & 13 \\
\hline Tree in bud & 18 & 13 \\
\hline Consolidation & 4 & 2 \\
\hline Cavity & 13 & 9 \\
\hline Ground glass opacity & 6 & 5 \\
\hline Pleural effusion & 5 & 6 \\
\hline Miliary nodules & 0 & 2 \\
\hline lymphadenopathy & 13 & 10 \\
\hline Bronchiectasis & 15 & 53 \\
\hline $\begin{array}{l}\text { Parenchymal } \\
\text { calcification }\end{array}$ & 0 & 13 \\
\hline Emphysema & 0 & \\
\hline
\end{tabular}

\section{Discussion}

Out of the 100 patients included in the study, 65 were found to be males 35 females. Most of the patients were males. In this study most of the patients were in the age group 51-60. In the study conducted by Hatipoglu et al. the mean age of the patient were 44 .

Regarding the HRCT findings in our study, finding of centrilobular nodules have a significant $\mathrm{p}$ value of 0.000010 . Hence in this study, centrilobular nodules and sputum positivity has a statistically significant association. In our study, centrilobular nodules were present in $63 \%$ sputum positive cases and are in close agreement with the study conducted by Tayfun Caliskan et al., where centrilobular nodules were present in $66 \%$ of cases. ${ }^{4}$

Regarding the HRCT pattern of "tree in bud", $\mathrm{P}$ value was found to be highly significant (0.000040).Hence in the present study "tree in bud" pattern and sputum positivity has a statistically significant association. "Tree in bud" pattern were present in $60 \%$ of patients with sputum positivity in our study whereas in the study conducted by Hatipoglu et al., "tree in bud pattern were present in $71 \% .^{5}$

In our study, cavitary lesions were present in $43 \%$ sputum positive cases with a statistically significant $\mathrm{p}$ value of 0.0007 . So in this study HRCT finding of cavity and sputum positivity has got a statistically significant association. The study carried out by Dr. Soujanya Bolla et al. in close agreement with the present study as cavitary lesions were present in $41.6 \%$ in his study. ${ }^{6}$

In the present study consolidation was present in only $13 \%$ of cases with sputum positivity and $p$ value is 0.04 , statistically significant association present with HRCT finding of consolidation and sputum positivity. In the study conducted by Im JG et al., consolidations was present in $52 \%$ of cases 6 . So our study is not in agreement with that study. May be because most of the cases in our study were reinfection cases.

Ground glass opacities were present in $20 \%$ of patients with sputum positivity in the present study with a $P$ value of 0.0596 . Since the $p$ value is $>0.05$, there is no statistically significant association with the finding of ground glassing and sputum positivity. in the study carried out by Tayfun Caliskan, ground glass opacities were present in $26 \%$ of cases with a $\mathrm{P}$ value of 0.9234 and is in close agreement with the present study.

In our study, mediastinal lymphadenopathy was present in $43 \%$ of cases with sputum positive pulmonary tuberculosis and a $\mathrm{P}$ value of 0.0015 . So there is a statistically significant association with the finding of mediastinal lymphadenopathy and sputum positivity. Mediastinal lymphadenopathy was present in $31 \%$ of cases with sputum positivity in the study conducted by Im JG et $\mathrm{al}^{7}$. 
In our study, sputum positive pleural effusion were present in $45.5 \%$ of cases with a $\mathrm{P}$ value of 0.235 . There is no statistically significant association between pleural effusion and sputum positivity in the present study. In the study conducted by Hatipoglu et al., pleural effusion was present in only $6 \%$ of cases 5 and is not in agreement with our study.

Bronchiectasis was present in $22 \%$ of sputum positive patients with a significant $\mathrm{P}$ value of 0.01 . This finding is in agreement with the study conducted by Tayfun Caliskan et al., in which bronchiectasis was present in $28 \%$ of cases.4 Other HRCT findings detected in this study include miliary nodules, parenchymal calcification and emphysema. None of these findings were noted in patients with sputum positivity.

To conclude, in our study, HRCT findings of centrilobular nodules, "tree in bud" pattern, cavity, consolidation, mediastinal lymphadenopathy and bronchiectasis have got a statistically significant association with sputum positivity. Our study is more in agreement with the study conducted by Tayfun Caliskan et al. ${ }^{4}$

\section{Conclusion}

This prospective observational study was conducted to evaluate the role of High Resolution Computed Tomography in the evaluation of active pulmonary tuberculosis and to find out the association between HRCT findings of active pulmonary tuberculosis and sputum positivity. HRCT findings like centrilobular nodules, "tree in bud", cavitation and mediastinal lymphadenopathy are the best parameters of active pulmonary tuberculosis. There is statistically significant association between these findings and sputum positivity, pointing out that these are the important things to be noted and mentioned while looking into the HRCT image of a patient suspected to be having active pulmonary tuberculosis.

\section{Conflict of Interest}

No conflict of interest

\section{References}

1. World Health Organization. Global tuberculosis report 2012. Geneva, Switzerland: World Health Organization Press; 2012:1272.

2. Treatment of Tuberculosis: Guidelines, fourth ed., WHO/HTM/TB/2009.

3. J.Foulds, R. O'Brien, New tools for the diagnosis of tuberculosis: the perspective of developing countries, Int. J. Tuberc. Lung Dis. 2 (1998) 778-783

4. Tayfun Caliskan, Tuncer Ozkisa et al. High resolution computed tomography findings in smear-negative pulmonary tuberculosis patients according to their culture status: 10.3978/j.issn.2072-1.

5. Hatipoglu ON, Osma E et al. HRCT findings in pulmonary tuberculosis. Thorax 1996; 51:397- 402.439.2014.03.41

6. Dr. Soujanya Bolla*, Dr. Chhaya Bhatt et al. Role of HRCT in Predicting Disease Activity of Pulmonary Tuberculosis. Gujarat Medical Journal / August-2014 Vol. 69 No.2.

7. Im JG, Hoi H et al. Pulmonary tuberculosis: CT findings- early active disease and sequential change with anti tuberculosis therapy. Radiology-1993:186:653-660 